\title{
Applications of Mindfulness-Based Interventions in School Settings: an Introduction
}

\author{
Joshua C. Felver $^{1} \cdot$ Patricia A. Jennings ${ }^{2}$
}

Published online: 22 December 2015

(C) Springer Science+Business Media New York 2015

\section{Introduction}

Mindfulness as a general topic of scientific inquiry has emerged as an increasingly popular topic for social scientists, educators, and psychologists in the last 30 years. To illustrate this growing interest, one need only search for peer-reviewed publications using "mindfulness" as the primary search term. Figure 1 displays publications in academic journals that were retrieved using PsychINFO and "mindfulness" as the keyword. As of November 2015, over three thousand scientific articles have been published $(n=3,350)$, with about one third of these articles having been published just in the last 2 years alone. What is also striking about the data displayed in this graph is not just the sheer volume of the work that is emerging, but also that the research appears to be growing at an exponential rate, suggesting an accelerating interest in the field among scholars. Indeed, given that so much time and energy is being devoted to the study of mindfulness, researchers have proposed the creation of new disciplines entirely devoted to the subject, coining such terms as contemplative science (Wallace 2006) and mindfulness psychology (Felver et al. 2013), and an entire journal (i.e., Mindfulness) is now devoted solely to the topic. However, although the Western scientific inquiry into the construct labeled as mindfulness has clearly established itself as a general domain in social science, there

Joshua C. Felver

jcfelver@syr.edu

1 Department of Psychology, College of Arts and Sciences, Syracuse University, 430 Huntington Hall, Syracuse, NY 13244, USA

2 CISE Department, Curry School of Education, University of Virginia, 206D Bavaro Hall, PO Box 400273,

Charlottesville, VA 22904, USA remains ample opportunity to investigate how mindfulness can be applied and studied within specific subsamples and in specific settings.

One such domain that has begun to receive increasing attention from the scholarly community is the application of mindfulness with youth in educational settings. Indeed, of all the studies collected in the aforementioned search, only $8 \%(n=256)$ have involved youth under the age of 18 , and only $1 \%(n=36)$ focused on youth in school settings using the keyword search terms "school," "education," or "classroom." Further, among the 256 articles that have focused on mindfulness and youth, the vast majority (79\%) have been published in just the last 5 years alone $(2011-2015, n=203)$. Mindfulness research has often been described as being in its infancy, and if this is the case, then the study of mindfulness with youth and in schools is in the prenatal stage of development.

This Special Section was developed to focus and channel the enthusiasm for applying mindfulness-based interventions into school settings by inviting leading researchers in the field to contribute their most recent work. This Special Section includes an exciting collection of cutting-edge research, including reviews of the existing work to date, outcomes from mindfulness interventions with students and teachers, and several other papers related to the topic.

Two reviews of the existing literature are included in this special issue. Feagans-Gould et al. (2016) gathered 48 articles and explored the defining characteristics and implementation details of mindfulness- and yoga interventions in school settings. Felver et al. (2016) selected 28 studies that implemented mindfulness-based intervention in school settings, and coded and analyzed study characteristics across multiple domains. These reviews not only provide a snapshot of the strengths and limitations in the research conducted to date, they also provide important and timely recommendations for future research into the topic. 
Fig. 1 Publications in academic journals with keyword "mindfulness"

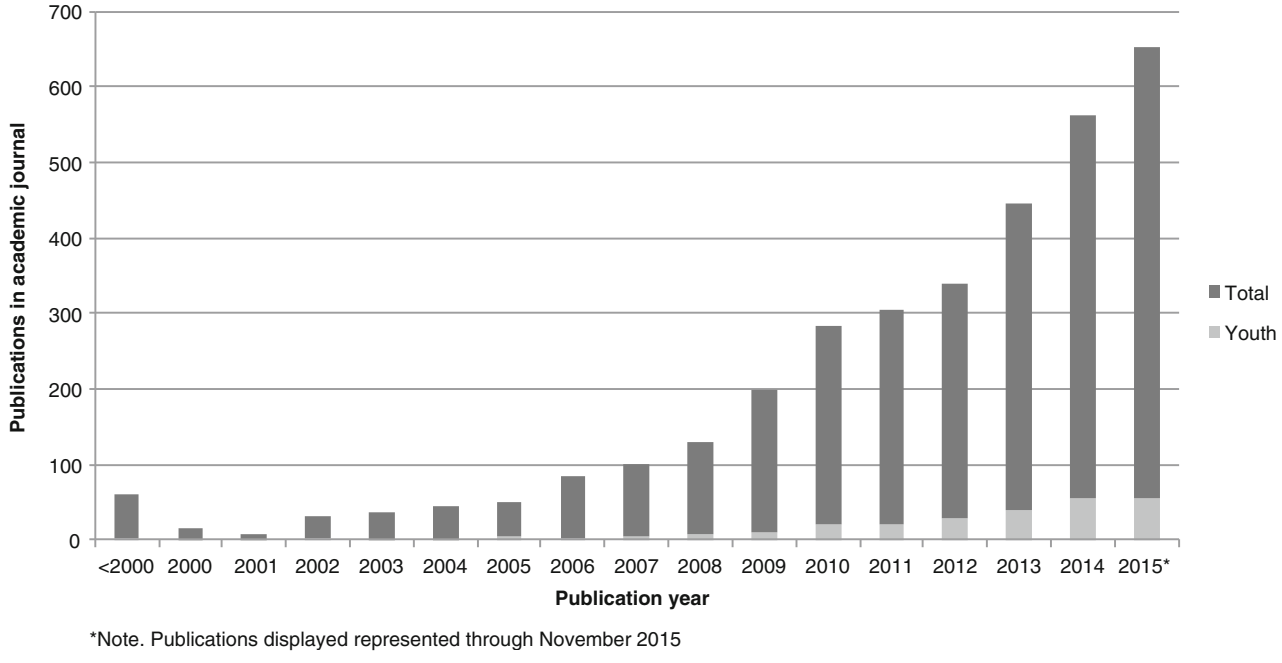

Six articles in this special issue demonstrate mindfulnessintervention effects and positive gains for youth in school settings across multiple age levels. Poehlmann-Tynan et al. (2016) explored the feasibility and the effects of an intervention with preschoolers from low socio-economic status backgrounds, and found that it is not only possible to implement mindfulness-based curricula with this population but that such experiences may benefit student attentional and self-regulatory skills. Bakosh et al. (2016) conducted their intervention study in an elementary school setting and found that $10 \mathrm{~min}$ of daily practice using pre-recorded instructions appeared to improve student academic achievement. Using a single subject, multiple baseline research design, Singh et al. (2016) similarly found that individual mindfulness training benefited student academic achievement in mathematics, and also that student's academic engagement improved following intervention. Dariotis et al. (2016) qualitatively investigated elementary student outcomes following intervention, and found that students and teachers were able to identify several important themes related to skills learning and application following training including the importance of coping strategies for stressful experiences and emotions. Bluth et al. (2016) investigated outcomes in an at-risk adolescent population, and found significant improvements in student mood following intervention. Bennett and Dorjee (2016) also explored the utility of mindfulness-based programming with adolescents, and their results suggest benefits to student mood and academic achievement.

Considering that the review by Felver et al. (2016) yielded only 28 studies that explored the utility of mindfulness-based intervention with youth in school settings, it is notable that these six intervention studies contained in this special issue represent a $21 \%$ increase in the total number of peer-reviewed articles on this particular topic. What is more important, however, is that these six studies address and provide the needed empirical evidence to support the utility of mindfulness-based interventions in school settings, including how such interventions benefit students' mental health and educational functioning, and how such interventions can be effectively delivered across a broad range of ages from pre-school to high-school. These results will be of interest to those seeking to implement similar programming for each of these age groups.

Three studies examined the benefits of mindfulness-based interventions for teachers. Taylor et al. (2016) found that teachers reported reduced stress following mindfulness training, and their data further elucidated some of the mechanisms which may be responsible for stress reduction. Schussler et al. (2016) similarly explored the mechanisms underlying effects following mindfulness training, and found that teachers' awareness of their internal states and improvements to their emotion-regulation may be contributing to reductions in self-reported stress. Harris et al. (2016) found that a 16-week brief, daily mindfulness and yoga program improved teachers' mindfulness, positive affect, classroom management, distress tolerance, physical symptoms, blood pressure, and protected against a blunting of the cortisol awakening response found among control group teachers at the end of the school year. These papers provide exciting evidence that mindfulness-based interventions can support teacher wellbeing and functioning, further extending the research into this important topic, as teachers' experience alarmingly high-rates of stress and burnout.

In addition to review and intervention papers, this special issue also includes methodological and theoretical contributions. Frank et al. (2016) contribute an important methodological paper reporting on the development and validation of a measure of mindfulness in teaching, providing a muchneeded instrument for those interested in conducting research in this field. Gueldner and Feuerborn (2016) highlight the utility of integrating mindfulness-based activities into the existing domain of social-emotional learning, suggesting interesting 
avenues for future program development and research. Finally, Jennings (2016) provides a commentary on the issues related to applying mindfulness-based activities in school settings addressing the issue of secularity and offering recommendations for best practices in public educational settings.

The knowledge represented in this Special Section offers insights into how the technology of mindfulness can be utilized in educational settings to the betterment of teachers and students; however, as with any new field of scientific inquiry, there remain several important questions that have yet to be addressed and explored. There are as yet no systematic studies on what the necessary recommended "dosage" of mindfulness practice is to effect change, nor is there any empirical data to inform how best to developmentally tailor mindfulness practices for younger populations. Indeed, there is no consensus on what the defining characteristics of a "mindfulness practice" even are for any population, thus preventing the necessary component analyses needed to elucidate the mechanisms of action behind these interventions. There have been no studies to date exploring the best modality for teaching mindfulness practices to youth (e.g., by a teacher, by an outside long-term mindfulness practitioner), and although it is recommended that instructors have a substantial personal practice of their own to deliver these interventions, there are currently no data supporting this recommendation. The long-term outcome studies on how mindfulness-based interventions affect development and academic achievement have also not been conducted, and as a result we do not know how these practices affect youth over time and as they age. We offer these suggestions as an illustration of the work and discoveries that lay ahead for future researchers interested in this topic, as indeed there are many questions to be answered, and undoubtedly more exciting questions to be asked as this research continues to flourish.

In conclusion, this special section captures the current state of the field, provides several timely studies that advance the study of mindfulness in educational settings, and greatly contributes to the existing research on this nascent topic of scientific inquiry. Indeed, using the aforementioned data obtained from PsychINFO, the 13 articles in this special issue represent a $36 \%$ increase in the existing studies on mindfulness-based interventions with youth in school settings. For researchers, educators, and clinicians interested in applying mindfulness-based interventions to support youth development, schools offer a unique and exciting setting for intervention application, and the articles presented in this special issue provide more of the necessary evidence to support further application and inquiry within this setting.
Compliance with Ethical Standards The manuscript does not contain clinical studies or patient data.

Ethical Approval This article does not contain any studies with human participants or animals performed by any of the authors.

Conflict of Interest The authors declare that they have no competing interests.

\section{References}

*References marked with an asterisk indicate manuscripts included in this special section.

*Bakosh, L. S., Snow, R. M., Tobias, J. M., Houlihan, J. L., \& BarbosaLeiker, C. (2016). Miximizing mindful learning: mindful awareness intervention improves elementary school students' quarterly grades. Mindfulness. doi:10.1007/s1267-015-0387-6.

*Bennett, K., \& Dorjee, D. (2016). The impact of a mindfulness-based stress reduction course (MBSR) on well-being and academic attainment of sixth-form students. Mindfulness. doi:10.1007/s12671-0150430-7.

*Bluth, K., Campo, R. A., Pruteanu-Malinici, S., Reams, A., Mullarkey, M., \& Broderick, P. C. (2016). A school-based mindfulness pilot study for ethnically diverse at-risk adolescents. Mindfulness. doi:10. 1007/s12671-014-0376-1.

*Dariotis, J. K., Mirabal-Beltran, R., Cluxton-Keller, F., Feagans Gould, L., Greenberg, M. T., \& Mendelson, T. (2016). A qualitative evaluation of student learning and skills use in a school-based mindfulness and yoga program. Mindfulness.

*Feagans Gould, L., Dariotis, J. K., Greenberg, M. T., \& Mendelson, T. (2016). Assessing fidelity of implementation (FOI) for school-based mindfulness and yoga interventions: a systematic review of the literature. Mindfulness. doi:10.1007/s12671-015-0395-6.

*Felver, J. C., Celis-DeHoyos, C., Tezanos, K., \& Singh, N. N. (2016) A systematic review of mindfulness-based interventions for youth in school settings. Mindfulness. doi:10.1007/s12671-015-0389-4.

*Frank, J. L., Jennings, P. A., \& Greenberg, M. T. (2016). Validation of the mindfulness in teaching scale. Mindfulness. doi:10.1007/ s12671-015-0461-0.

*Gueldner, B., \& Feuerborn, L. L. (2016). Integrating mindfulness-based practices into social and emotional learning: a case application. Mindfulness. doi:10.1007/s12671-015-0423-6.

*Harris, A. R., Jennings, P. A., Katz, D. A., Abenavoli, R. M., \& Greenberg, M. T. (2016). Promoting stress management and wellbeing in educators: outcomes of the CALM intervention. Mindfulness. doi:10.1007/s12671-015-0451-2.

*Jennings, P. A. (2016). Mindfulness-based programs and the American public school system: Recommendations for best practices to ensure secularity. Mindfulness. doi:10.1007/s12671-015-0477-5

*Poehlmann-Tynan, J., Vigna, A. B., Weymouth, L., Gerstein, E. D., Zabransky, M., Lee, P., \& Zahn-Waxler, C. (2016). A pilot study of contemplative practices with economically disadvantaged preschoolers: children's empathic and self-regulatory behaviors. Mindfulness. doi:10.1007/s12671-015-0426-3.

*Schussler, D., Jennings, P. A., Sharp, J., \& Frank, J. (2016). Improving teacher awareness and well-being through CARE: a qualitative analysis of the underlying mechanisms. Mindfulness. doi:10.1007/ s12671-015-0422-7. 
*Singh, N. N., Lancioni, G. E., Karazsia, B. T., Felver, J. C., Myers, R. E., \& Nugent, K. (2016). Effects of Samatha meditation on active academic engagement and math performance of students with attention deficit/ hyperactivity disorder. Mindfulness. doi:10.1007/s12671-015-0424-5.

*Taylor, C., Harrison, J., Haimovitz, K., Oberle, E., Thompson, K., Schonert-Reichl, K., \& Roeser, R. W. (2016). Examining potential mechanisms of stress reduction in a mindfulness-based intervention for school teachers: results from a randomized-control trial. Mindfulness. doi:10.1007/s12671-015-0425-4.

Felver, J. C., Doerner, E., Jones, J., Kaye, N., \& Merrell, K. M. (2013). Mindfulness in school psychology: applications for intervention and research. Psychology in the Schools, 50, 531-547.

Wallace, B. A. (2006). Contemplative science: where Buddhism and neuroscience converge. New York: Columbia University Press. 